\title{
Control of Prestimulus Activity Related to Improved Sensory Coding within a Discrimination Task
}

\author{
Takashi Yoshida ${ }^{1,3}$ and Donald B. Katz ${ }^{1,2,3}$ \\ ${ }^{1}$ Department of Psychology, ${ }^{2}$ Program of Neuroscience, and ${ }^{3}$ Volen Center for Complex Systems, Brandeis University, Waltham, Massachusetts 02454
}

Network state influences the processing of incoming stimuli. It is reasonable to expect, therefore, that animals might adjust cortical activity to improve sensory coding of behaviorally relevant stimuli. We tested this hypothesis, recording single-neuron activity from gustatory cortex (GC) in rats engaged in a two-alternative forced-choice taste discrimination task, and assaying the responses of these same neurons when the rats received the stimuli passively. We found that the task context affected the GC network state (reducing betaand gamma-band field potential activity) and changed prestimulus and taste-induced single-neuron activity: before the stimulus, the activity of already low-firing neurons was further reduced, a change that was followed by comparable reductions of taste responses themselves. These changes served to sharpen taste selectivity, mainly by reducing responses to suboptimal stimuli. This sharpening of taste selectivity was specifically attributable to neurons with decreased prestimulus activities. Our results suggest the importance of prestimulus activity control for improving sensory coding within the task context.

\section{Introduction}

Neural networks are intrinsically active, and the activity observed just before sensory stimulation can affect subsequent sensory responses (Arieli et al., 1996; Castro-Alamancos, 2002; Petersen et al., 2003; Fiser et al., 2004; Murakami et al., 2005; Fontanini and Katz, 2006; Curto et al., 2009) (for review, see Haider and McCormick, 2009), in agreement with modeling studies showing that preexisting states help determine how neurons within a network respond to input (Martí et al., 2008; Miller and Katz, 2010).

If random, uncontrolled fluctuations of network state add "noise" to sensory responses, it is also likely that awake animals actively control network states dependent on behavioral context. Such control could play a key role in flexible sensory processing, adapting the information contained in sensory responses to task demands (Desimone and Duncan, 1995; Arieli et al., 1996; Gilbert and Sigman, 2007; Fontanini and Katz, 2008; Haider and McCormick, 2009) and potentially explaining context-dependent modulation of neural responses (Fanselow and Nicolelis, 1999; Otazu et al., 2009). Thus, an understanding of the interactions between preexisting network states and behaviorally relevant sensory responses - such as those produced during a sensory discrimination task to gain reward-will be crucial for deeper understanding of sensory function.

Taste is an ideal system for this investigation. Anticipation of taste stimuli modulates prestimulus activity in gustatory cortex (GC) (Yamamoto et al., 1988; Ohgushi et al., 2005; Stapleton et al., 2006, 2007; Gutierrez et al., 2010). In human functional magnetic reso-

Received Aug. 20, 2010; revised Jan. 4, 2011; accepted Jan. 13, 2011.

This work was supported by National Institute on Deafness and Other Communication Disorders Grant DC007703 (D.B.K.), The Uehara Memorial Foundation in Japan (T.Y.), and Japan Society for the Promotion of Science (T.Y.).

Correspondence should be addressed to either of the following: Takashi Yoshida, Department of Medicine, Kyushu University, 3-1-1 Maidashi, Higashi-ku, Fukuoka, Japan 812-8512, E-mail: yoshidac57bl6@gmail.com; or Donald B. Katz, Department of Psychology, Program of Neuroscience, and Volen Center for Complex Systems, Brandeis University, 415 South Street, Waltham, MA 02454, E-mail: dbkatz@brandeis.edu.

DOI:10.1523/JNEUROSCI.4380-10.2011

Copyright $\odot 2011$ the authors $\quad 0270-6474 / 11 / 314101-12 \$ 15.00 / 0$ nance imaging (fMRI) studies, cognitive priming via expectation and attention impacts taste processing in several areas including GC (Nitschke et al., 2006; Grabenhorst and Rolls, 2008). Furthermore, correlations between spontaneous firing and taste responses have been observed in both dog and rat GC (Funakoshi and Ninomiya, 1983; Yamamoto et al., 1989). GC responses, which represent multiple properties of taste stimuli (Yamamoto et al., 1988, 1989; SmithSwintosky et al., 1991; Ito and Ogawa, 1994; Stapleton et al., 2006) in distinct epochs of time-extensive responses (Katz et al., 2001a) can be modulated by arousal (Fontanini and Katz, 2005, 2006; Tort et al., 2010), satiety (de Araujo et al., 2006) (but also see Rolls et al., 1988), and learning (Yamamoto et al., 1989; Accolla and Carleton, 2008; Grossman et al., 2008; Gutierrez et al., 2010). As of yet, however, there have been few examinations relating context-dependent alterations of prestimulus activity — such as those occurring in a task requiring discrimination of tastes - to the specifics of subsequent taste responses.

Here, we address this issue, directly comparing GC activity in two behavioral contexts within single sessions - a taste discrimination task in which rats were required to discriminate tastes, and a "passive" context in which the same stimuli were presented passively without requirement of behavioral responses. Our analyses confirmed that both prestimulus and poststimulus GC activity were affected by behavioral context, and that the modifications of activity before stimulus during the task were related to the modulation of taste responses, resulting in enhanced information available for stimulus identification, specifically in the period preceding behavioral responses.

\section{Materials and Methods}

Animals

Female Long-Evans rats were our subjects (280-350 g; Charles River). All experimental procedures followed the guidelines of Brandeis University Institutional Animal Care and Use Committee. Dur- 
ing experiment, rats were maintained on water deprivation (30 min/d access postsessions).

\section{Taste discrimination task (task)}

We trained rats on a two-alternative forced-choice (2afc) task in which two taste solutions were associated with each choice. Two hundred millimolar $\mathrm{NaCl}(\mathrm{N}), 500 \mathrm{~mm}$ sucrose (S), 15 or $40 \mathrm{~mm}$ citric acid (C) (15 mm in three and $40 \mathrm{~mm}$ in two rats), or $2 \mathrm{~mm}$ quinine- $\mathrm{HCl}(\mathrm{Q})$ were used as stimuli ( $40 \mathrm{~mm}$ citric acid solutions were used for the first two rats, but as this $\mathrm{C}$ concentration proved too high for subsequent several rats to allow habituation, these rats' data were discarded, and a $15 \mathrm{~mm} \mathrm{C}$ solution was used in the remaining three rats). Three nose poke ports were set in one wall of an operant chamber (see Fig. 1A). After a light-emitting diode (LED) signaled trial availability, a rat was required to hold its nose in the center port for $0.75-1.5 \mathrm{~s}$ (time randomized across trials) to trigger delivery of $20 \mu \mathrm{l}$ of either N, S, C, and Q, through either a lick spout or an intraoral cannula (IOC) (a small bundle of tubes inserted into a guide tube surgically implanted into the oral cavity); delivery order was pseudorandom-all four tastes were presented once every four trials. The rat was then required to remove its nose from the center port and to poke one of the two side ports. If the rat poked the side port associated with that taste, water was delivered as a reward. The next trial started after intertrial interval (ITI) of either at least $15 \mathrm{~s}$ (correct trials) or $20 \mathrm{~s}$ (incorrect trials). N and Q were consistently associated with one side, and $\mathrm{S}$ and $\mathrm{C}$ with opposite side; thus, the choice could not be made on the basis of palatability ( $\mathrm{N}$ and $\mathrm{S}$ are palatable, whereas $\mathrm{C}$ and $\mathrm{Q}$ are aversive). Associated sides were counterbalanced among rats. For initial (presurgical) training, solutions were presented through short lick spouts attached to each port. During recording sessions, each solution was presented through an IOC. Solutions were ejected from the IOC by nitrogen pressure under control of solenoid valves (Katz et al., 2001a). All behavioral apparati were controlled by custom-coded programs (LabVIEW; National Instruments).

\section{Training protocol for taste discrimination task}

Training sessions were between 45 and 60 min long, 5-6 d per week.

Habituation and shaping of nose poking. Rats were handled and habituated to the experimental chamber at least for $3 \mathrm{~d}$ during which the three ports were covered and inaccessible. During the shaping of nose poking, one of three ports was made accessible. After an LED signaled trial start, poking and holding of rat's snout inside the port through a delay period caused the delivery of $20 \mu \mathrm{l}$ of water through a tube attached to the bottom of the port, followed by the extinction of the LED. The delay period was set at $0.2 \mathrm{~s}$ for the first session and was gradually extended $(0.2$ $\mathrm{s}$ steps, incremented every 15-30 water deliveries) until it reached $1.5 \mathrm{~s}$. After a $13 \mathrm{~s}$ ITI, a $2 \mathrm{~s}$ period without nose poking started the next trial with the LED signal. This prevented rats from nose poking continuously during the ITI.

Shaping lasted for at least $3 \mathrm{~d}$, during which time the position of the port changed daily such that all three ports were used.

Discrimination task training. After shaping, training of a two-taste discrimination started. The initial taste pair was either $\mathrm{NaCl}$ versus sucrose or citric acid versus quinine, so that the discrimination could not be based on palatability. One taste was associated with each side port, an association that was maintained throughout the experiment. For the first few days of training, one of two side ports was covered, and only the center and one side port were used simultaneously (one-option forcedchoice trial). In each trial, the rat first needed to poke into center port for $0.75-1.5 \mathrm{~s}$ (precise value selected randomly in each trial) to obtain a taste solution, and then to move and poke into the opened port for water reward. One taste was delivered repeatedly; the taste and opened side were switched together every 20-30 trials.

After rats learned to move smoothly toward the side ports after taste consumption at the center port, both side ports were made accessible, and rats were trained to perform a $2 \mathrm{afc}$ task. In each trial, one of two tastes was chosen randomly, and water reward was delivered only after correct side poking. A $5 \mathrm{~s}$ time-out was added to the ITI after incorrect trials. Each session continued until 100 trials were completed or until $45 \mathrm{~min}$ had elapsed.
In the early phase of training, rats occasionally showed a response preference for one side independent of the taste stimuli. If a rat showed such a side preference for two consecutive sessions, in the next session the preferred port was covered, and the rat was forced to choose the other side (with repeated delivery of the associated taste) for 20-30 trials (i.e., one-option forced-choice trial), and then training paradigm was switched back to 2 afc task.

After performance reached asymptotic learning ( $>85 \%$ correct trials for three consecutive sessions or $80 \%$ for five nonconsecutive sessions), training repeated with the other taste pair, and only then was the full four-taste paradigm used-four tastes delivered in a pseudorandom order. Training continued until performance once again reached criterion, after which rats were given ad libitum access to water for at least $5 \mathrm{~d}$ before surgery. Training durations were varied across rats from 4 weeks to several months.

Postsurgery training. Postsurgery training started at least $10 \mathrm{~d}$ after surgery. IOC (a manifold of thin cannulae) and tubes for taste delivery were connected to a surgically implanted guide tube; rats were habituated to movements under the connection of tubes inside the experimental box with the three ports covered for several days. The water deprivation schedule was then restarted. After habituation, rats were trained to the taste discrimination task with four tastes following the same protocol before surgery except for the taste delivery method-tastes were now delivered directly into the oral cavity through the IOC. When behavioral performance reached a criterion of $70-75 \%$ correct trials/session for at least three sessions, recording session started. Passive blocks were added to the sessions, occurring before or after the training session at least three sessions before recording sessions started. Headstages and cables for electrophysiology were connected and habituated for a couple of sessions before the recording.

\section{Passive delivery task (passive)}

For assays of passive responses, the three ports were covered and inaccessible. Tastes and water were then delivered pseudorandomly via IOC under freely moving conditions without any predictive cue. The interstimulus interval was $25 \mathrm{~s}$. Although this constant interval could conceivably facilitate a rat's anticipation of taste delivery, reliable activity changes (e.g., ramping or transient firing) were seldom (if ever) observed just before stimulus delivery (data not shown), suggesting that GC activity was not affected by the constant interval.

\section{Surgery}

After reaching learning criterion ( $80-85 \%$ correct), rats were anesthetized with a mixture of ketamine $(100 \mathrm{mg} / \mathrm{kg})$, xylazine $(5 \mathrm{mg} / \mathrm{kg})$, and acepromazine $(1 \mathrm{mg} / \mathrm{kg})$, and a bundle of 16 wires equipped with a microdrive (Katz et al., 2001b) was implanted unilaterally (in two rats) or bilaterally (in three rats) into GC (anteroposterior, $+1.4 \mathrm{~mm}$; mediolateral, $\pm 5 \mathrm{~mm}$; dorsoventral, $-4.4 \mathrm{~mm}$ from bregma and pial surface) as described previously (Katz et al., 2001a; Fontanini and Katz, 2005). Guide tubes for IOC were inserted from the side of maxillary first molar to the side of skull, and stabilized with dental acrylic. Rats were given ad libitum access to water for at least $10 \mathrm{~d}$ after surgery, during which time antibacterial ointment and penicillin were applied.

\section{Recording sessions}

A daily session consisted of one task and one passive block separated by 5-10 min. Sessions lasted $\sim 1 \mathrm{~h}$, during which each taste was delivered at least 8 times (usually 10 to 15 times delivery) in each block. Passive blocks were added before or after task blocks. Recordings were performed once rats had reacquired asymptotic performance ( $>70 \%$ correct). Neural signals were amplified, filtered [gain, 100; filter, $154-8.8 \mathrm{~K} \mathrm{~Hz}$ for spike; and gain, 1000; filter, $0.7-170 \mathrm{~Hz}$ for local field potential (LFP) data; PBX-257; Plexon], and digitized with a Multichannel Acquisition Processor system (sampling frequency, $40 \mathrm{~K}$ and $1 \mathrm{~K} \mathrm{~Hz}$ for spike and LFP; Plexon). For spike recording, all waveforms that crossed a preset amplitude threshold were stored.

Spike sorting was performed off-line with commercially available software (Offline Sorter; Plexon). Stored waveforms were plotted in a two- or three-dimensional space based on distinctive waveform features (the first three principal components, peak and valley amplitudes, amplitude of a 
specific time point of waveforms, or nonlinear energy). Distinct, nonoverlapping clusters of waveforms were labeled; a cluster was considered to be derived from a single unit only when (1) interspike intervals below a refractory period $(1 \mathrm{~ms}$ ) made up $<0.05 \%$ of all waveforms in the cluster, and (2) the valley (or peak) amplitudes showed approximately normal distribution without truncation by the threshold of waveform detection. Most (i.e., all but 16 of 118 taste responsive) neurons were recorded from distinct electrode wires. Electrodes were lowered $80 \mu \mathrm{m}$ after each recording sessions, to ensure that different populations of neurons were isolated in each session. After the last recording session (1-10 sessions per rat), electrolytic lesions were made by passing current through the electrodes, and recording sites were confirmed by histology. Neural activity was recorded mainly from dysgranular insular, but also from granular and agranular insular (see Fig. 1D,E).

\section{Data analyses}

Data and statistical analyses were performed by custom programs coded in Matlab (The MathWorks) and SPSS (SPSS, Inc./IBM). $\alpha$ level was set at $5 \%$ for all analyses, with Bonferroni's corrections for multiple comparisons as necessary.

To evaluate interactions between context and neural variables, we performed multiple-factor repeated-measures ANOVAs (RM-ANOVAs). Significant interactions between context and other factors were followed by simple main effect analyses to evaluate the context effect on specific levels of the factors. For within-neuron comparisons between contexts (i.e., paired and repeated-measures analyses), neurons with missing values (e.g., 0 firing rates) were excluded from analyses (the sample size of each analysis is described in either the text or figure legends). In this study, we used both parametric tests (ANOVA) and nonparametric tests (specifically, Wilcoxon's signed rank and rank sum tests). Parametric tests assume normal distributions, and since firing rates (and other normalized values) often fail to satisfy this assumption, nonparametric tests may sometimes be the better option. However, we did use ANOVA to examine interaction effects between factors, so that we could avoid the strongly restrictive significance levels required when performing multiple independent comparisons. The signed rank test with Bonferroni's correction resulted in very similar conclusions to those observed using ANOVA (see Figs. 2, 5B, $C, 7 D-F$ ). Error bars indicate SEMs in figures. As rats produced few errors per taste in these sessions, only trials in which rats correctly responded were used for analyses of task data.

\section{Behavior quantification}

Reaction time (RT) was defined as the duration between taste delivery and the rat's exit from the center port immediately before entry into a side port. Data from sessions in which $<75 \%$ of the trials were responded to correctly were discarded from the analysis, as were trials in which RTs were more or less than three times the SD in each taste.

\section{LFP}

LFP power was computed using a multitaper method in the Chronux Matlab toolbox (http://chronux.org/). Data from trials contaminated by slow large deflections (presumably artifact) were discarded by visual inspection (sessions in which fewer than seven trials in each taste were delivered were discarded). After removal of $60 \mathrm{~Hz}$ line noise, power spectra were computed for the $0.5 \mathrm{~s}$ epoch preceding stimulus delivery and the $0.3-0.8 \mathrm{~s}$ epoch after stimulus in each trial ("mtspectrumc"; time-band width product: TW $=3$; number of tapers: $K=5$ ), and single trials were averaged for each taste and pooled across recording sites, recording sessions, and rats (total $n=214$ recording sites). This poststimulus epoch ( +0.3 to +0.8 s) minimized possible contamination from evoked LFP components [the "gustatory evoked potential" (GEP)] (Tort et al., 2010). For statistical analysis, mean LFP power in beta (15-30 $\mathrm{Hz})$, low-gamma $(40-50 \mathrm{~Hz})$, and high-gamma $(70-85 \mathrm{~Hz})$ frequency ranges were computed at each recording site, transformed into decibels (in which the power was distributed relatively normally), and evaluated with ANOVA.

In this study, raw LFP power, rather than data normalized by prestimulus baseline (a procedure that reduces the effect of across-session and individual variability), was used: the main purpose of this study was to examine the effect of context on both prestimulus and poststimulus activity, and the very changes of prestimulus power that we sought to analyze make it complicated to evaluate poststimulus power normalized to baseline. To evaluate between-session and between-rat variability, therefore, we analyzed LFP power in each individual rat (via context/ taste/session ANOVAs). Of five rats, most (three or four, depending on the conditions) showed significant effects that matched the group means (i.e., lower power during task at $15-30$ and $40-50 \mathrm{~Hz}$ in prestimulus epoch and at $40-50$ and $70-85 \mathrm{~Hz}$ in poststimulus epoch) (see Fig. 2). Although LFP power was indeed variable across sessions, the reported directions of effects appeared to be consistent in many cases. These suggest that results of raw LFP power reported here were robust to session/ individual variability.

To analyze evoked LFP components that were time-locked to taste delivery [the GEP (Tort et al., 2010)], digitized voltage signals around taste delivery (from -500 to $+800 \mathrm{~ms}$ ) were low-pass filtered (cutoff frequency, $30 \mathrm{~Hz}$ ) by fourth-order Butterworth filter (Matlab function "butter"), averaged across trials for each taste and context, and pooled across channels and experimental sessions. Data contaminated by artifacts were discarded by visual inspection (e.g., larger deflection, sudden offset, etc.). Because the polarity of the GEP (i.e., positive or negative) varied depending on the recording sites, the highest amplitude of either the peak or valley of the short-latency (from +20 to $+200 \mathrm{~ms}$ after stimulus) response, measured relative to baseline (mean amplitude across a $500 \mathrm{~ms}$ window before stimulus delivery) was used for statistical analysis. If any 15 consecutive samples $(15 \mathrm{~ms}$ ) around the highest amplitude were over three times the SD of $500 \mathrm{~ms}$ prestimulus epoch, this value was defined as a significant GEP. For between-context comparison, all data for which significant GEPs were obtained at least in one context were used. If the highest amplitude was peak (or valley) amplitude, amplitudes of peaks (or valleys) were compared between contexts.

\section{Firing rate modulation}

Firing rates were computed in the prestimulus $(0.5 \mathrm{~s}$ before taste delivery), and poststimulus epochs (taste delivery to $0.2 \mathrm{~s}$ before movement start), a value that varied across trials. In passive, the average value of poststimulus epoch was used for the poststimulus time window for each taste.

Significant taste responses were identified by comparing prestimulus and poststimulus firing rates ( $p<0.05$, Wilcoxon's signed rank test) for each taste in each context. The number of tastes that elicited significant responses in each context was used for the evaluation of tuning properties [(see Figs. $6 A, 7 D$, an analysis of side-port association information (i.e., N-Q or S-C pair), data not shown]. A neuron was defined as a taste-responsive neuron if the response was significant to at least one taste in either task or passive. A modulation index (MI), defined as $\left(\mathrm{FR}_{\text {task }}-\mathrm{FR}_{\text {passive }}\right) /\left(\mathrm{FR}_{\text {task }}+\mathrm{FR}_{\mathrm{pas}}\right.$ sive), where $\mathrm{FR}_{\text {task }}$ and $\mathrm{FR}_{\text {passive }}$ are mean firing rates in task and passive, respectively, was used to quantify context-related differences in firing. Absolute size of firing rate difference may be important. However, firing rates in GC neurons range from a few hertz to tens of hertz, and "highfrequency" neurons tend to bias results lacking normalization. The normalization method (e.g., modulation index) was used to avoid this bias. Neurons that failed to fire action potentials in either context were removed from the modulation index analysis. For analyses of poststimulus taste response modulations, significant responses were pooled across tastes and analyzed together for the simplicity of presentation (see Figs. $4 C, D, 7 A-C$ ). As described in Results, taste response modulation was also analyzed in each taste separately, and the tendency of weak taste responses to decrease during task was observed ubiquitously.

\section{Response variability}

The Fano factor, a ratio of the variance to the mean of spike counts across trials, was computed using a $0.2 \mathrm{~s}$ window shifted by $0.05 \mathrm{~s}$ steps around particular events (i.e., the port entry and taste delivery). If the mean spike count in a bin was 0 , that bin was excluded from the analysis. For statistical analysis, Fano factors were averaged across bins in preevent $(0.5 \mathrm{~s}$ before event) and postevent epochs ( $0.5 \mathrm{~s}$ for the center port entry and $1 \mathrm{~s}$ for taste delivery), and pooled across neurons; ANOVA was applied to each event separately. For within-neuron comparisons between contexts, neurons for which the Fano factor had no value (i.e., 0 spike count across 
trials across bins) in at least one epoch in either context were excluded (86 and 82 neurons were used for the entry and taste delivery epochs). Because a three-way ANOVA (taste/context/time) revealed no significant main effect of taste, Fano factors were averaged across tastes.

Trial-to-trial correlation between prestimulus and poststimulus activity

Spike counts from epochs $0.5 \mathrm{~s}$ before and $1 \mathrm{~s}$ after stimulus were normalized [(each trial value - mean of trials)/SD of trials] separately for each taste, and then pooled together across tastes in each context. To minimize any possible artifacts caused by long-term trends across a session, trials in each taste of each context were divided into first and second halves, and the normalization was performed in each half. Finally, the correlation between prestimulus and poststimulus epochs was computed for each neuron.

\section{Taste selectivity}

To evaluate taste selectivity, sharpness and strength indexes were computed, based on mean firing rates from the poststimulus epoch [sharpness: Rainer et al. (1998); strength: Freedman et al. (2006)]. Sharpness was defined as $\left(n-\sum \mathrm{FR} i / \mathrm{FR}_{\text {best }}\right) /(n-1)$, where $\mathrm{FR} i$ is mean firing rate of each taste $(i=1-4), \mathrm{FR}_{\text {best }}$ is the maximum firing rate among tastes, and $n$ is total number of stimuli $(n=4)$. A sharpness of 1 indicates that a neuron responded to one taste, and the value 0 indicates equal responses across tastes. Strength was defined as $\left(\mathrm{FR}_{\text {best }}-\mathrm{FR}_{\text {worst }}\right) /\left(\mathrm{FR}_{\text {best }}+\right.$ $\left.\mathrm{FR}_{\text {worst }}\right)$, where $\mathrm{FR}_{\text {best }}$ and $\mathrm{FR}_{\text {worst }}$ are maximal and minimal mean firing rates among tastes, respectively. In the sharpness and strength index analyses, neurons that failed to respond to any tastes (i.e., firing rates of 0 ) were removed.

\section{Response classification}

Classification analysis was based on that used previously (Foffani and Moxon, 2004). In this analysis, only simultaneously recorded ensembles containing at least four neurons were used (total 14 ensembles containing 4-11 neurons). Firing rates computed from the $1 \mathrm{~s}$ after taste delivery in each trial were normalized by the maximum firing rate of the neuron. A single trial ensemble response was defined as a "test" vector, to be compared with "template" vectors for each taste made from the average of the remainder of the trials. Euclidean distances between the test and template vectors for each taste were computed, and the test vector was classified to the taste in which this distance was smallest. Classification was repeated for all trials, and the percentage of correctly classified trials was determined. To evaluate the side-port association (or palatability)related information, the scores that the test vector was classified to the side-port-associated pair (N-Q or S-C pair, or N-S or C-Q pair in the case of palatability) was also computed.

We also evaluated the effect of block order [i.e., whether task preceded passive (task $\rightarrow$ passive) or vice versa (passive $\rightarrow$ task)] on all analyses described above. The vast majority of these tests came back negative (by multiple-factor ANOVA including a factor of the block order). Although block order had occasional effects - on the magnitude of LFP power (related to Fig. 2) and on the magnitude of the MI (MI-post) difference between pre-high and pre-low neurons (related to Fig. $7 A-C$ ) - the context-dependent differences still existed in most conditions of both cases (see Results) (see Table 1). Thus, block order does not explain the context-dependent modulation described below.

\section{Results}

\section{Rats can discriminate tastes}

To examine whether encouraging proper stimulus identification affects cortical taste processing, we recorded single-unit activity and LFPs in two behavioral contexts: a taste discrimination task (task) and passive taste delivery (passive). In task, three ports for nose poking were available in an experimental box (Fig. $1 \mathrm{~A}$, left). In each task trial, a rat first obtained one of four tastes solutions: $\mathrm{NaCl}(\mathrm{N})$ (salty), sucrose (S) (sweet), citric acid (C) (sour), or quinine $\mathrm{HCl}(\mathrm{Q})$ (bitter), at the center port; tastes were delivered through an IOC (a manifold of thin tubes threaded from the headcap into the oral cavity) $0.75-1.5 \mathrm{~s}$ after entrance into the
A Task

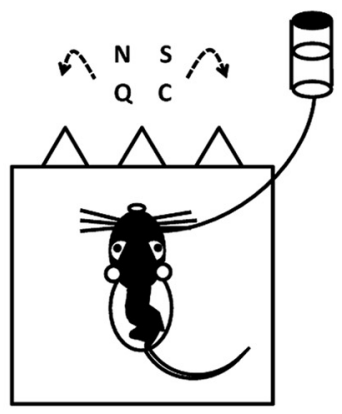

B
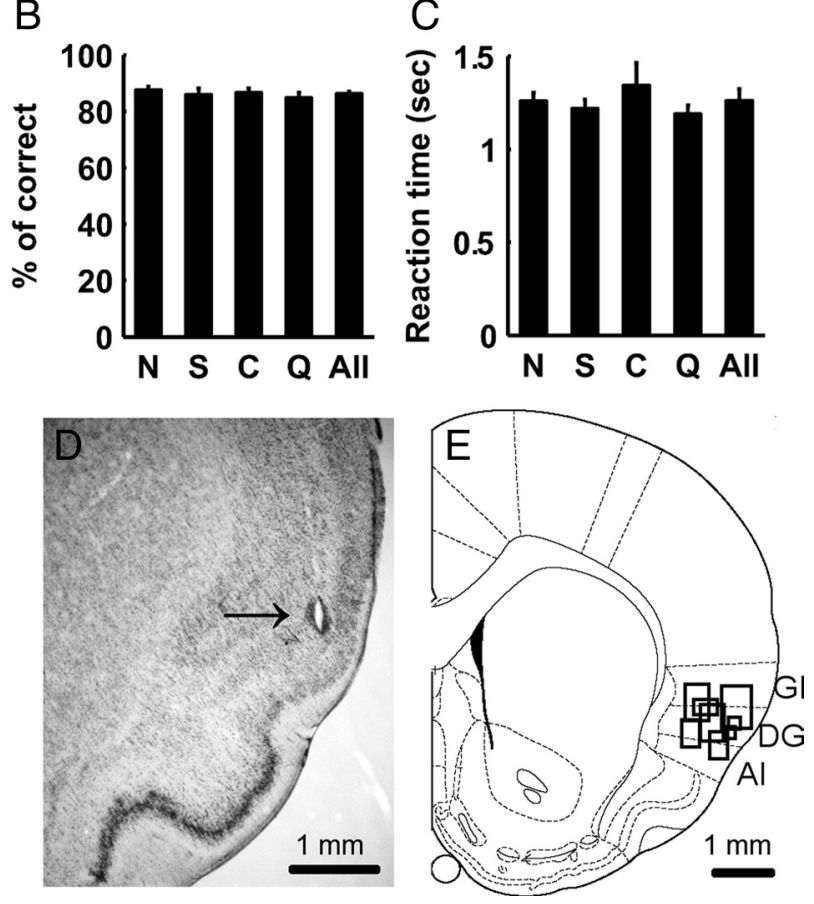

Figure 1. Behavioral contexts and task performance. $\boldsymbol{A}$, Schematic diagrams of the taste discrimination task (task; left) and the passive taste delivery (passive; right). In an experimental box, three ports for nose poking (triangles) were set. In task, rats obtained pseudorandomly selected taste solutions by nose poking the center port (N, NaCl; S, sucrose; $\mathrm{C}$, citric acid; $\mathrm{Q}$, quinine), and then were required to poke the side port associated with the delivered taste stimulus to obtain water reward. In passive, the ports were covered and the taste solutions were presented pseudorandomly, without nose poking on the part of the rat. In both contexts, the taste solutions and water were delivered directly to the rat's oral cavity through a manifold of thin tubes inserted into the implanted cannula (see main text for details). $\boldsymbol{B}, \boldsymbol{C}$, Task performance $(\boldsymbol{B})$ and reaction time $(\boldsymbol{C})$ are independent of the taste type $(n=28$ sessions from 5 rats; $p>0.1$ by one-way ANOVA). All, Average of across tastes. Error bars indicate SEM. $\boldsymbol{D}, \boldsymbol{E}$, Recording areas. $\boldsymbol{D}$, Nissl-stained image of a recording site (arrow). An electrolytic lesion was made by passing current through electrode wires after the conclusion of the experiment. $\boldsymbol{E}$, The black boxes indicate all recording areas, reconstructed by electrolytic lesions and tracks of electrodes, taking into account the numbers of recording sessions (electrodes were moved ventrally $\sim 80 \mu \mathrm{m}$ after each recording). Tips of electrode bundles were spread over several hundred micrometers $(<500 \mu \mathrm{m})$. Activity was recorded mainly from dysgranular insular cortex (DG), but also from granular (GI) and agranular insular (Al) cortex. For simplicity of presentation, all recording areas are projected on one hemisphere, but recordings were performed bilaterally in three rats. Schematic diagram was reprinted with publisher's permission from Paxinos and Watson (1998).

port (time chosen randomly). The rat was required to discriminate taste types by moving to the proper side port- - two tastes (N and Q) were associated with the one side, and other two (S and C) with the other side (Fig. 1A, left) such that liked (N and $\mathrm{S}$ ) and disliked (C and $\mathrm{Q}$ ) tastes were balanced between two sides; water reward was obtained only after a move to the correct port. 
A
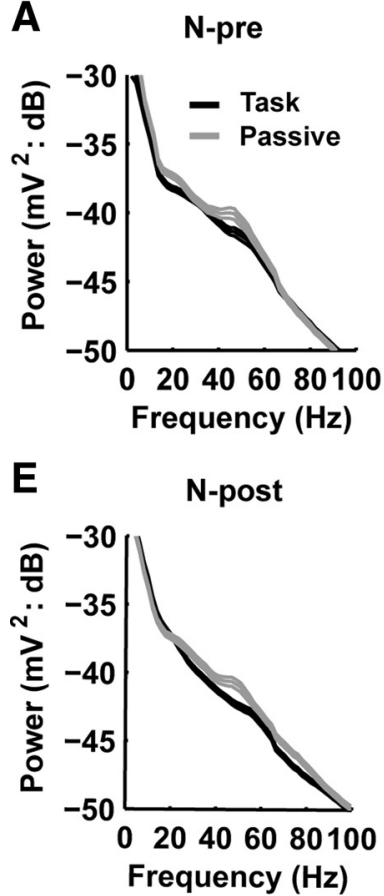

B $\quad 15-30 \mathrm{~Hz}$
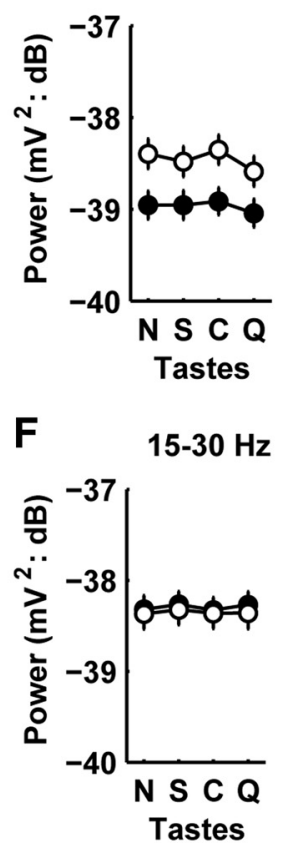

C $\quad 40-50 \mathrm{~Hz}$
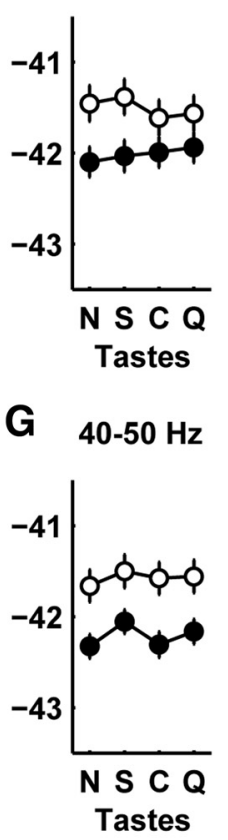

\section{I}
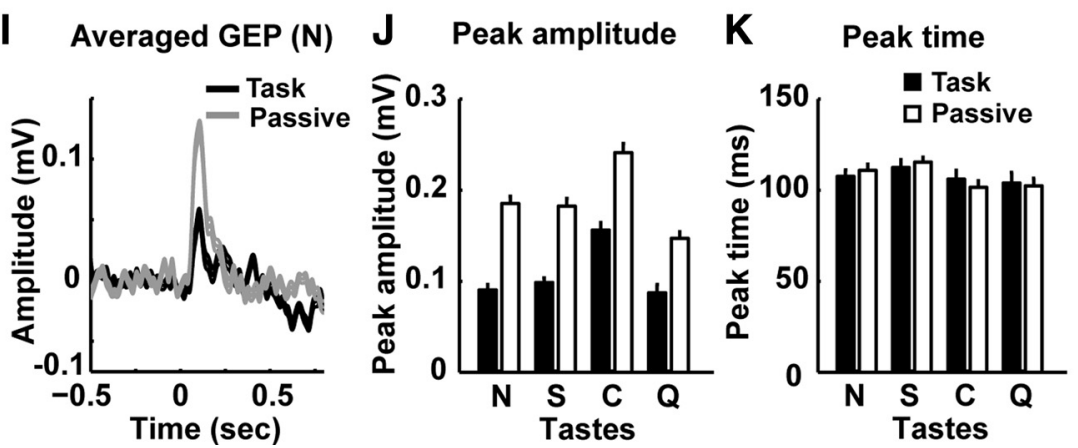

Figure 2. Behavioral context modifies LFP. $\boldsymbol{A}, \boldsymbol{E}$, Averaged power spectra of all recording sites ( $n=214$ recording sites) before ( $0.5 \mathrm{~s}$ period from -0.5 to $0.0 \mathrm{~s})(\boldsymbol{A})$ and after $(0.5 \mathrm{~s}$ period from +0.3 to $+0.8 \mathrm{~s})(\boldsymbol{E}) \mathrm{NaCl}$ delivery. Power was lower in the beta and gamma frequency ranges in both prestimulus and poststimulus epochs of task (black lines). The three lines indicate mean and mean \pm SE. $\boldsymbol{B}-\boldsymbol{D}$, $\boldsymbol{F}-\boldsymbol{H}$, Averaged power in beta $(15-30 \mathrm{~Hz})(\boldsymbol{B}, \boldsymbol{F})$, low-gamma $(40-50 \mathrm{~Hz})(\boldsymbol{C}, \boldsymbol{G})$, and high-gamma $(70-85 \mathrm{~Hz})(\boldsymbol{D}, \boldsymbol{H})$ frequency ranges before $(\boldsymbol{B}-\boldsymbol{D})$ and after stimulus $(\boldsymbol{F}-\boldsymbol{H})$. In task, LFP power was significantly lower than in passive at most frequencies. $\boldsymbol{B}, \boldsymbol{G}, p<0.05$ for main effects of context and taste by $2 \mathrm{~W}$-RM-ANOVA. $\boldsymbol{C}, \boldsymbol{H}, p<0.05$ for simple main effect of context in all tastes after significant contexttaste interaction. $\boldsymbol{D}, p<0.05$ for simple main effect of context in $\mathrm{N}$ (but not other tastes) after significant context-taste interaction. $\boldsymbol{F}$, No significant difference between contexts. N, NaCl; S, sucrose; C, citric acid; $Q$, quinine. I, Averaged GEP in $\mathrm{NaCl}(\mathrm{N})$ trials $(n=133$ recording sites) in task (black lines) and passive (gray, mean and mean \pm SE). The GEP amplitude was smaller in task.J, Between-context comparison of the peak amplitudes of the GEP (filled bars, task; open bars, passive). N = 133, 127, 104, and 76 for N, S, C, and Q, respectively. Only the data containing significant peaks or valleys at least in one context ( $>3$ times the SD of 500 ms before stimulus epoch) (also see Materials and Methods) were used. Amplitudes in task were significantly lower than those in passive for all tastes (all values of $p<0.05$ by Wilcoxon's signed rank test with Bonferroni's correction). The difference between contexts was not affected by the block order in most conditions: for 8 comparisons - 4 per taste $\times 2$ possible orders - the task versus passive difference failed to reach significance in only one ( $Q$ in passivefirst sessions). $\boldsymbol{K}$, Between-context comparison of peak times of the GEP (filled bar, task; open bar, passive). $N=133,127,104$, and 76 in N, $\mathrm{S}, \mathrm{C}$, and Q. There was no difference between contexts for any taste, nor any effect of block order. Error bars indicate SEM.

Neural activity was assayed in well trained animals [average of $86 \pm 4 \%($ mean $\pm \mathrm{SD})$ correct] $($ Fig. $1 \mathrm{~B})$, in which performance and RT (the time elapsing between taste delivery and movement onset; average, $1.26 \pm 0.34 \mathrm{~s}$ ) were independent of specific taste stimulus ( $p>0.1$ by one-way ANOVA) (Fig. 1 B, C). Although rats can discriminate taste palatability within a few hundred milliseconds in certain circumstances (Halpern and Tapper, 1971), RTs observed here were comparable with those observed in similar types of taste discrimination tasks (MacDonald et al., 2009; Gutierrez et al., 2010), despite differences in taste delivery methods (IOC vs licking).
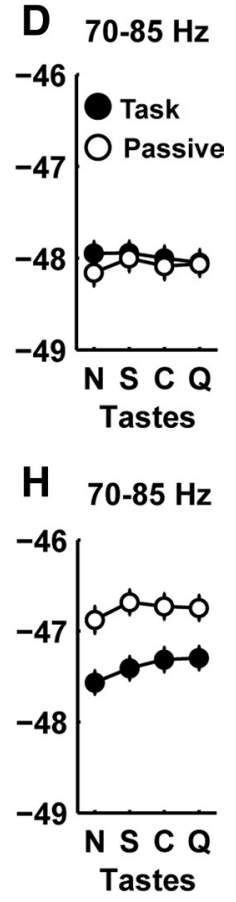

Although in task rats approached the port to process taste stimuli actively and discriminatively, they received the same stimuli without warning and without any behavioral demands in passive (IOC delivery, although less natural than licking, allowed us to present solutions in the absence of voluntary behavior such as approaching and nose poking). For passive sessions, which occurred immediately before or after task sessions, ports were inaccessible (Fig. $1 A$, right).

\section{Behavioral context modulates local field potentials}

To examine whether behavioral context affects GC network state, we analyzed LFPs (for recording sites, see Fig. 1D,E), which reflect coherent activities of population of neurons (i.e., network activity) and the power spectra of which provide a useful clue to how neurons in networks are working together (Buzsáki and Draguhn, 2004). Averaged LFP power spectra calculated for the periods before and after $\mathrm{NaCl}$ delivery are shown in Figure 2, $A$ and $E$, respectively. LFP power in the $40-50 \mathrm{~Hz}$ range (lowgamma) was higher in passive than in task during both epochs (Fig. 2C, $G$ ), as was prestimulus beta $(15-30 \mathrm{~Hz}$ ) (Fig. $2 B$ ) and poststimulus high-gamma $(70-85 \mathrm{~Hz})$ (Fig. $2 \mathrm{H}$ ) activity [all differences were significant at $p<0.05$ by two-way repeatedmeasures ANOVA (2W-RM-ANOVA)]. No context differences were detected in prestimulus high-gamma activity (save $\mathrm{N}$, $p<0.05$, simple main effect of context after significant context-taste interaction by $2 \mathrm{~W}$-RM-ANOVA) (Fig. $2 D$ ) or in poststimulus beta activity (Fig. $2 F$ ). In general, LFP power was lower when the passive block preceded the task block than when task preceded passive (data not shown), the context-dependent modulations described above were mostly robust (Table 1): context changed the GC network state during the period preceding administration of stimuli, differences that persist into the period in which the stimuli are processed.

We also examined short-latency LFP modulations that were time-locked to taste delivery [GEP (Tort et al., 2010)]. GEP amplitudes were significantly lower during task ( $p<0.05$ by Wilcoxon's signed rank test with Bonferroni's correction) (Fig. $2 I-K$ ), as has also reported for auditory evoked potentials in rat primary auditory cortex (Otazu et al., 2009). This result suggests that the task context suppresses input or direct response to input to GC.

Behavioral context modifies single neuron activity To examine whether these distinct behavioral/network conditions affect single-neuron firing, we analyzed the activity of GC single neurons recorded in both task and passive. A total of 203 
Table 1. Block order effects on between-context modulations of LFP power

\begin{tabular}{|c|c|c|c|c|c|c|c|c|}
\hline \multirow[b]{2}{*}{ Frequency/block order } & \multicolumn{4}{|c|}{ Prestimulus epoch } & \multicolumn{4}{|c|}{ Poststimulus epoch } \\
\hline & N & $S$ & C & 0 & N & $S$ & C & 0 \\
\hline \multicolumn{9}{|l|}{$15-30 \mathrm{~Hz}$} \\
\hline All & L & L & L & $\mathrm{L}$ & NS & NS & NS & NS \\
\hline $\mathrm{T} \rightarrow \mathrm{P}$ & L & L & L & L & NS & $\underline{H}$ & NS & NS \\
\hline $\mathrm{P} \rightarrow \mathrm{T}$ & L & $\underline{\text { NS }}$ & L & L & NS & $\underline{L}$ & NS & NS \\
\hline \multicolumn{9}{|l|}{$40-50 \mathrm{~Hz}$} \\
\hline All & L & $\mathrm{L}$ & $\mathrm{L}$ & $\mathrm{L}$ & $\mathrm{L}$ & $\mathrm{L}$ & $\mathrm{L}$ & $\mathrm{L}$ \\
\hline$T \rightarrow P$ & L & $\mathrm{L}$ & $\mathrm{L}$ & $\mathrm{L}$ & $\mathrm{L}$ & L & $\mathrm{L}$ & $\mathrm{L}$ \\
\hline $\mathrm{P} \rightarrow \mathrm{T}$ & L & $\mathrm{L}$ & $\mathrm{L}$ & $\mathrm{L}$ & NS & NS & $\mathrm{L}$ & NS \\
\hline \multicolumn{9}{|l|}{$70-85 \mathrm{~Hz}$} \\
\hline All & $\mathrm{H}$ & NS & NS & NS & $\mathrm{L}$ & $\mathrm{L}$ & $\mathrm{L}$ & L \\
\hline $\mathrm{T} \rightarrow \mathrm{P}$ & $\mathrm{H}$ & NS & NS & NS & L & L & $\mathrm{L}$ & L \\
\hline $\mathrm{P} \rightarrow \mathrm{T}$ & $\mathrm{H}$ & $\underline{\mathrm{H}}$ & $\underline{\mathrm{H}}$ & NS & $\mathrm{L}$ & $\mathrm{L}$ & $\mathrm{L}$ & L \\
\hline
\end{tabular}

Block order effect was analyzed by ANOVA (order by context by taste) followed by simple main-effect analysis. As a whole, LFP powers in the passive $\rightarrow$ task order ( $\mathrm{P} \rightarrow \mathrm{T}:$ passive preceded task) were lower than those in the task $\rightarrow$ passive $\operatorname{order}(\mathrm{T} \rightarrow \mathrm{P}$ : task preceded passive) independent of context, reflecting the significant main effects of block order in all frequency ranges ( $p<0.05$ by RM-ANOVA) (data not shown). However, the context-dependent modulations were consistent in most conditions, although the block order did affect a subset of the conditions (e.g., the $70-85 \mathrm{~Hz}$ power in prestimulus epoch and the $40-50 \mathrm{~Hz}$ power in poststimulus epoch). All, All sessions including both block order shown in Figure 2. N, NaCl; S, sucrose; C, citric acid; $\mathrm{Q}$, quinine. H and L indicate higher and lower power during task-that is, either a significant main context effect (with no interaction) or interaction followed by simple main effect of context in each taste and order $(p<0.05)$. NS, No significance. Underline in each symbol indicates different result of the analysis of sessions broken down according to order.
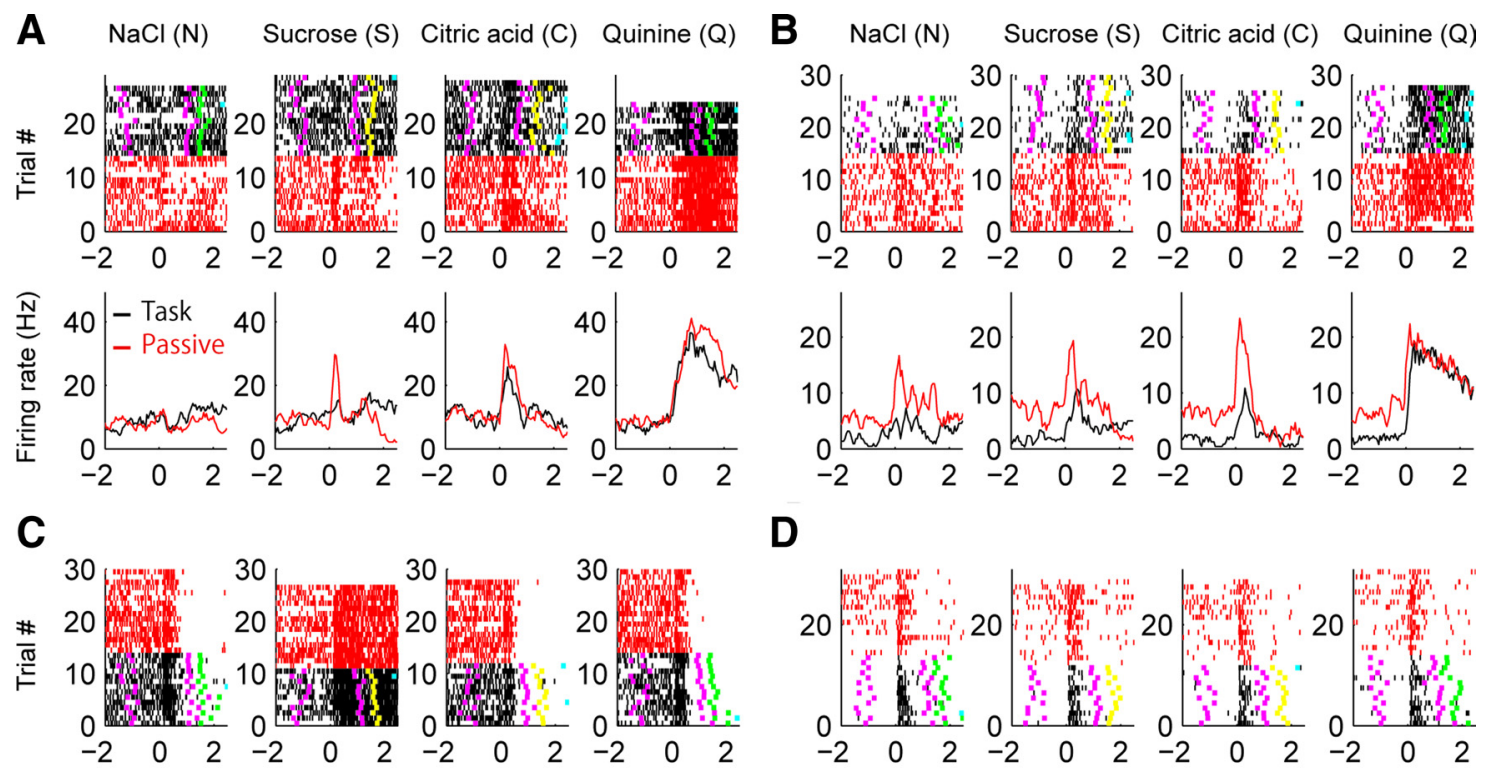

D
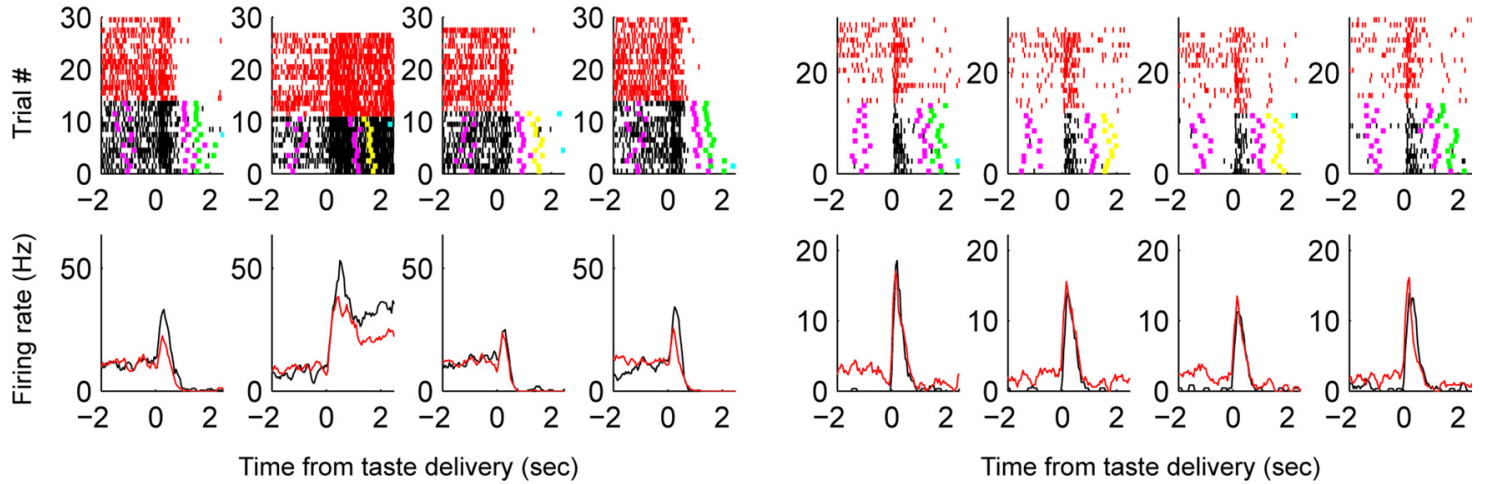

Figure 3. Behavioral context modifies single-unit activities. Examples of taste-responsive neural firing during task (black) and passive (red). Raster graphs (top panels) indicate action potential timings (vertical hash marks) in individual trials (each row) relative to the taste delivery (at $0 \mathrm{~s}$ ); the colored bars represent event timing during task [magenta before stimulus, center port entry; magenta after stimulus, movement start; green and yellow, right and left port entry; light blue, reward delivery (located outside the figures in many trials)]. Peristimulus time histograms (bottom panels) were computed with $0.2 \mathrm{~s}$ sliding windows $(0.05 \mathrm{~s}$ steps). The neurons shown in $\boldsymbol{A}$ and $\boldsymbol{B}$ produced weak taste responses that were suppressed in task (i.e., to $\mathrm{N}, \mathrm{S}$, and $\mathrm{C}$ ) and strong taste responses that were comparable between contexts (to $\mathrm{Q}$ ). The neuron shown in $\mathrm{C}$ produced taste responses that increased in task. Prestimulus activity was modified in the neurons shown in $\boldsymbol{B}$ and $\boldsymbol{D}$.

neurons was recorded; of these, 118 neurons were "tasteresponsive" in that their firing rates changed significantly in response to at least one taste in either task or passive $(p<0.05$ by Wilcoxon's signed rank test). Additional analyses were restricted to these taste-responsive neurons.

As expected from the LFP analysis, we found that context modulated neural firing in both prestimulus and poststimulus epochs. Examples of context-dependent taste response modulations are shown in Figure 3. The neuron shown in Figure $3 A$ responded to $Q$ strongly, and to C and S weakly. The strong Q response was comparable in task and passive, whereas the smaller response to $S$ was suppressed during task. The effect of context on the neuron shown in Figure $3 B$ was similar (this weak-response reduction was the typical pattern) (see below), but for this neuron, both prestimulus firing and sensory responses were affected. In contrast, the responses of the neuron shown in Figure $3 C$ were stronger in task. Another example of prestimulus modulation, this time in the absence of a task effect on taste responses, is shown in Figure 3D. 

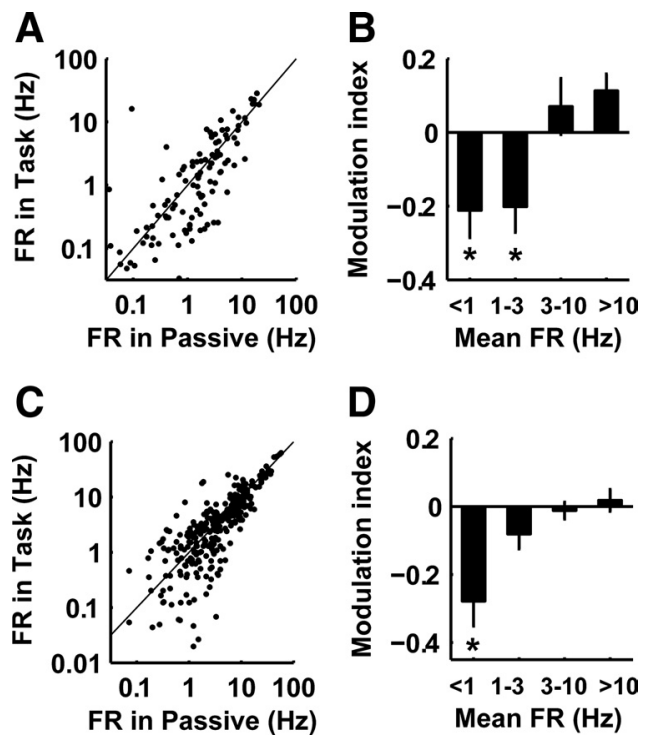

Figure 4. Prestimulus and poststimulus activity is modified by task in a firing ratedependent manner. $\boldsymbol{A}$, A between-context comparison of prestimulus firing. Each dot indicates the mean prestimulus firing rate (FR) $(0.5 \mathrm{~s}$ period before stimulus delivery) for each tasteresponsive neuron. Means were computed for all trials across tastes in each context. Neurons with 0 firing rates were excluded for purposes of log scale plotting, but included in the statistical firing rate comparison ( $n=118$ ). $\boldsymbol{B}$, The Mls of low firing frequency neurons were significantly less than 0 , indicating the reduction of prestimulus activity in task. Asterisks indicate $p<0.05$ by Wilcoxon's signed rank test with the Bonferroni's correction ( $n=45,38,24$, and 10 from left to right; total $N=117$ neuron - one neuron was removed for lack of firing in either context). $\mathrm{Ml}$ in prestimulus epoch was computed using the average firing rates for all tastes from each neuron. $\mathbf{C}$, Comparison of firing rate of taste responses between contexts. All significant taste responses pooled across four tastes ( $n=319$ taste responses; $n=81,84,81$, and 73 responses in $N, S, C$, and Q, respectively) are plotted, save for 0 firing rate cases. $D$, The modulation indices of low-amplitude taste responses were significantly less than 0 . The asterisk indicates $p<0.05$ by Wilcoxon's signed rank test with the Bonferroni's correction ( $n=64,94,105$, and 53 from left to right). Taste responses were pooled across tastes (total $n=316$ responses; 3 taste responses were removed for lack of firing). Error bars indicate SEM.

Although the grand, across-neuron average of prestimulus activity ( $0.5 \mathrm{~s}$ epoch just before stimulus) did not differ between task and passive (mean \pm SE: $3.3 \pm 0.5 \mathrm{~Hz}$ in task, $3.1 \pm$ 0.4 in passive; $p>0.1$ by Wilcoxon's signed rank test), a careful examination revealed that this grand mean masked effects in subsets of neurons; specifically, lower-firing neurons (i.e., those closer to the origin in Fig. 4A) mostly clustered below the diagonal (task $=$ passive) line, suggesting that the impact of context on prestimulus activity depended on basal firing rates. To pursue this apparent effect further, we computed a MI that quantified the difference between contexts for each neuron. This value was positively correlated with mean firing rates $(r=0.23 ; p<$ $0.05)$. When we subdivided groups of neurons according to mean firing rate, this result was confirmed: MIs in low-firing neurons were significantly lower than 0 ( $p<0.05$ by Wilcoxon's signed rank test with Bonferroni's correction) (Fig. $4 B$ ). Task further quieted the prestimulus firing of neurons with low spontaneous firing rates.

We performed identical analyses on the epoch between taste administration and $0.2 \mathrm{~s}$ before movement start ( $\sim 1 \mathrm{~s}$ duration) (Fig. 1C) to determine how taste responses were modulated by contexts. The mean firing rates for all significant responses, pooled across tastes, are plotted together in Figure $4 C$ (total $n=$ 319 responses from 118 neurons); overall, these taste responses were slightly lower in the task context $[5.8 \pm 0.48 \mathrm{~Hz}$ in task, $6.0 \pm 0.46 \mathrm{~Hz}$ in passive, median of difference: $-0.21 \mathrm{~Hz}, p<$
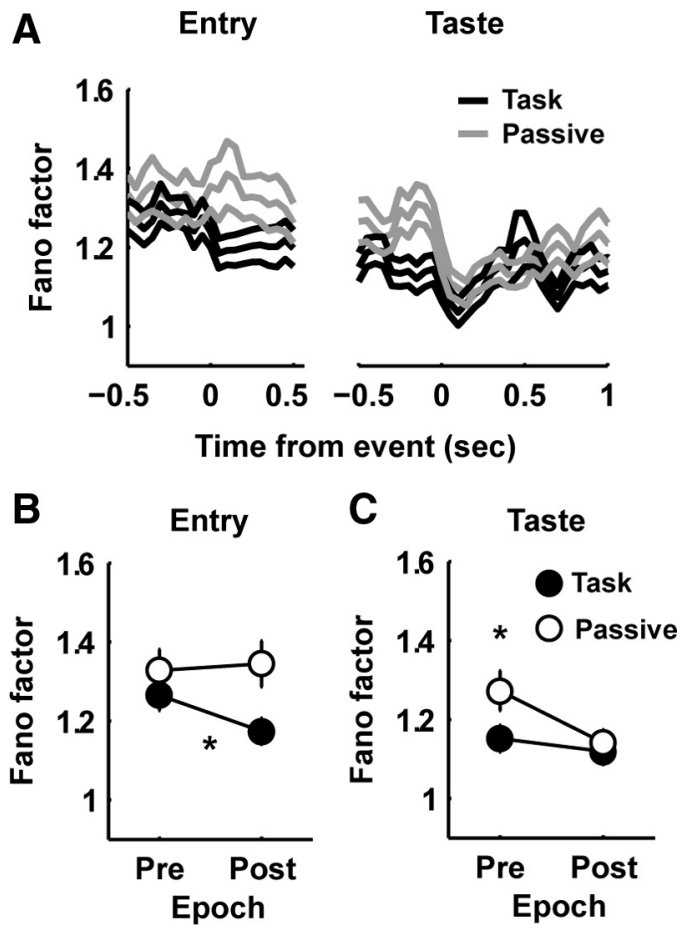

Figure 5. Trial-to-trial variability of neural activity is modified by task. $\boldsymbol{A}$, The Fano factor (trial-to-trial firing rate variability) decreases at the time of port entry in task (black lines in left); a similar reduction occurs at the time of stimulus delivery in passive (gray lines in right). The Fano factor was computed with $0.2 \mathrm{~s}$ moving window ( $0.05 \mathrm{~s}$ step). The three lines indicate mean and mean \pm SE. $\boldsymbol{B}$, In task, the Fano factor during the postentry epoch is significantly lower than that during the pre-entry epoch ( $n=86$; asterisk: $p<0.05$ by simple main effect of the epoch in task after significant context-epoch interaction). C, The Fano factor in task is significantly lower than that in passive during the prestimulus but not poststimulus epoch ( $n=$ 82; asterisk: $p<0.05$ by simple main effect of the context in pretaste delivery epoch after significant context-epoch interaction). Significant reductions between epochs were only observed in passive ( $p<0.05$ by simple main effect of epoch in passive).

0.05 by Wilcoxon's signed rank test (Fig. $4 C$ ), but no difference for each taste response (data not shown)]. This effect was specific to low-frequency responses; high firing taste responses appeared mostly unmodified by context change (Fig. 4C). This result was confirmed by analysis of MI: poststimulus MI was positively correlated with mean firing rate $(r=0.28, p<0.05$ for all responsive cases; $r$ ranged from 0.22 to 0.33 across tastes, $p<0.05$ in each case), and was significantly lower than 0 in low-frequency responses $(p<0.05$, Wilcoxon's signed rank test with Bonferroni's correction) (Fig. 4D). Thus, the changes in taste response magnitudes were also mostly inhibitory_-small taste responses were even smaller in task.

It has been suggested that trial-to-trial variability is another variable related to network function (Churchland et al., 2006, 2010). We therefore analyzed trial-to-trial variability before and after stimulus delivery using the Fano factor, the ratio of spike count variance to mean spike count. Figure $5 A$ reveals a reduction in firing variability after center port entry during the prestimulus period within task (the average timings of these events in relation to taste delivery were used to provide time references for comparison to passive data-these time points are "random" for passive data, referring to no specific events, and are identified only to highlight the epoch effect in task around the entry). Statistical evaluation confirmed the difference before and after center-port entry in task ( $p<0.05$ for simple main effect of epoch in task but not in passive after 2W-RM-ANOVA) (Fig. 5B). 
The difference between contexts vanished after stimulus delivery, as the Fano factor underwent a sudden stimulus-induced reduction in passive (Fig. $5 A$, right; $C$ ). Thus, it appears that GC activity is "stabilized" before stimulus delivery within task-a stabilization that somewhat reduces the size of, but not the variability of, sensory responses themselves (Fig. 4D).

\section{Task increases the selectivity of single-neuron sensory responses}

To examine the effect of these changes on taste coding, we analyzed how context affected single-neuron tuning properties. At the broadest level, our analysis suggests that taste response tuning was mostly unchanged by the switch between behavioral contexts. For $65 \%$ (77 of 118) of the taste-responsive neurons, the "best taste" - the stimulus that induced the strongest responsewas the same in task and passive. Some tunings did in fact change (35\% of the taste-responsive neurons), but firing patterns across tastes were highly correlated between the two contexts (median $r$, $0.82)$.

Although the "peak" responses of most GC neurons were maintained, their breadth of the responsiveness was not. Neurons responded more broadly in passive than task (Fig. 6A); the number of tastes inducing significant responses within task was lower than that observed in passive (mean \pm SE; $1.8 \pm 0.13$ in task, $2.2 \pm 0.13$ in passive; $p<0.05$, Wilcoxon's signed rank test). Accordingly, indices of response sharpness (a value of 1 indicating that the neuron responded to only one taste, and a value of 0 indicating that the neuron responded to all four tastes equally) and selectivity strength (normalized difference between best and worst responses) (see Materials and Methods) were significantly higher in task ( $p<0.05$, Wilcoxon's signed rank test) (Fig. $6 B, C)$. These results suggest that task sharpens the existing taste response tuning (almost certainly thanks to reductions of suboptimal responses) (Fig. 4D).

Sharpness and strength indices were also computed during the movement epoch $(+0.1$ to $+0.6 \mathrm{~s}$ after movement start $)$, and there was no significant difference between contexts in either index (data not shown). Furthermore, whereas previous studies have reported that information about side-port association (i.e., right or left) is represented in orbitofrontal cortex and GC under similar types of sensory discrimination tasks (Feierstein et al., 2006; Roesch et al., 2006; Furuyashiki et al., 2008; MacDonald et al., 2009), we found that only 6\% (7 of 118) and 5\% (6 of 118) neurons responded specifically to the port-associated pair of tastes (i.e., N-Q or S-C) in task and passive, respectively. Therefore, at least in our experiment, GC neurons represent little information about side-port association, if any, and task specifically impacted the activity before movement, rendering it more taste-selective.

\section{Modulation of taste responses in task is related to prestimulus activity changes}

We next tested whether task-related sensory and prestimulus modulations are linked. Initial analysis revealed that GC taste responses and prestimulus firing rates were correlated from trial to trial in both contexts-35\% (41 of 118 neurons) and 30\% (35 of 118) of taste-responsive neurons showed significantly positive correlations in task and passive, respectively. The means of the correlation coefficient distributions were significantly higher than 0 in both contexts (mean, 0.17 and 0.14 in task and passive; $p<0.05$ by $t$ test in both contexts).

Between-context modulations in prestimulus and poststimulus epochs were also correlated [i.e., MI in prestimulus and post- stimulus epoch (MI-pre and MI-post), $r=0.33, p<0.05$ (Fig. $7 A$ ). Note: This was affected by block order (see below)]. To further examine this point, we next divided our sample of tasteresponsive neurons into subgroups based on MI-pre; that is, we separately analyzed neurons with MI-pre $>0$ (pre-high; $n=47)$ and neurons with MI-pre $<0$ (pre-low; $n=70$ ) (one neuron was removed because it failed to fire during the prestimulus epoch). The mean MI-post was lower than 0 in pre-low neurons, whereas mean MI-post of pre-high neurons was slightly higher than 0 (Fig. $7 B, C)(p<0.05$, by Wilcoxon's signed rank test with Bonferroni's correction.). This main point, the fact that lower prestimulus firing in task context was followed by similarly reduced taste response magnitudes, occurred regardless of the block order [MI-post in pre-low: $-0.24 \pm 0.08$ in passive $\rightarrow$ task $(\mathrm{P} \rightarrow \mathrm{T})$ and $-0.19 \pm 0.04$ in task $\rightarrow$ passive $(\mathrm{T} \rightarrow \mathrm{P}) ; p<0.05$ in both cases], although context order did have some effect on the magnitude of MI-post in pre-high neuron [MI-post in pre-high: $-0.04 \pm 0.03$ (no significance, NS) in $\mathrm{P} \rightarrow \mathrm{T}$ and $0.12 \pm 0.03(p<0.05$ different from 0 ) in $\mathrm{T} \rightarrow \mathrm{P}$. The lower MI-post of pre-high in $\mathrm{P} \rightarrow \mathrm{T}$ was also reflected in the correlation between MI-pre and -post: $r, 0.19$ $(\mathrm{NS})$ in $\mathrm{P} \rightarrow \mathrm{T}$ and $0.37(p<0.05)$ in $\mathrm{T} \rightarrow \mathrm{P}]$.

These differences did not translate into a significant difference between the groups in the number of tastes responded to (although the difference between contexts was significant, $p<0.05$ by $2 \mathrm{~W}$-ANOVA) (Fig. 7D), but task-induced increases in sharpness and strength indices were attributable to pre-low neurons $(p<0.05$, simple main effect of context in pre-low neurons after significant context-neuron type interaction) (Fig. 7E,F). Together, these results suggest that task reduces the firing of a subset of neurons before stimulus delivery, neurons that then represent tastes more narrowly. A smaller subset of neurons conveys reliable taste information independent of context, but the overall effect is one of increased response specificity.

\section{Ensemble responses provide higher-quality taste information in task}

It is tempting to predict that task-induced modulations of network, prestimulus, and sensory responses exist to improve the quality of stimulus-related information provided by ensembles of cortical neurons. We tested this possibility, quantifying the efficacy with which taste responses of simultaneously recorded GC neurons specified which stimulus had been delivered.

The result of this analysis, restricted to simultaneously recorded ensembles containing $>3$ responsive neurons without regard to prestimulus firing $(n=14$ populations containing $4-11$ neurons), is shown in Figure 8. Figure $8 \mathrm{~A}$ shows the firing of one representative ensemble. Each row is one trial, each column is the taste response of a single neuron in the ensemble, and the brightness is response magnitude. Taste specificity is apparent in both the task (left) and passive (right) [e.g., neuron 1 responded to N, and neuron 5 to $S$; also obvious is the general response similarity between task and passive, and the sharpening of responses in task (e.g., neuron 7 responds more specifically to C)].

Each dot in Figure $8 B$ represents classification results in task and passive using one GC ensemble; the ensemble in Figure $8 A$ is indicated with an arrow. For most (10 of 14) of the ensembles, tastes were more reliably identified in task than passive (task, $69 \pm 4 \%$; passive, $60 \pm 5 \%$; $p<0.05$ by signed rank test). Although the differences appear relatively modest, they were striking given the fact that even in passive the average percentage correct was much higher than chance $(25 \%)$ - that is, the improvement was above and beyond the relatively high-quality classification that can be done with passive responses. In fact, for four 
A

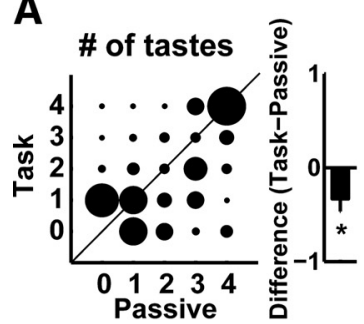

C

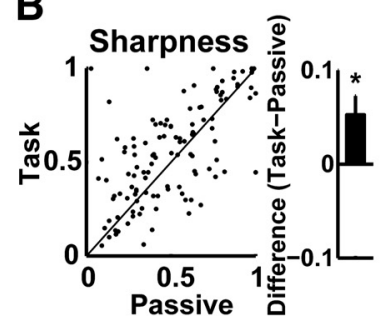

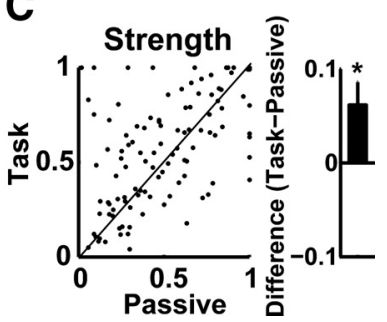

Figure 6. Taste selectivity increases in task. $\boldsymbol{A}$, The number of tastes which induce significant taste responses in each neuron is lower in task $(n=118)$. The sizes of circles indicate the number of neurons in a particular group. The biggest circle $([x, y]=[4,4])$ indicates 15 neurons, and the smallest circles (e.g., $[4,1])$ indicate 1 neuron. $\boldsymbol{B}, \boldsymbol{C}$, By both sharpness $(\boldsymbol{B})$ and strength $(\boldsymbol{C})$ indices, taste selectivity is higher in task ( $n=117 ; 1$ neuron was removed because it failed to fire in either context). The asterisks indicate $p<0.05$ by Wilcoxon's singed rank test. Error bars indicate SEM.

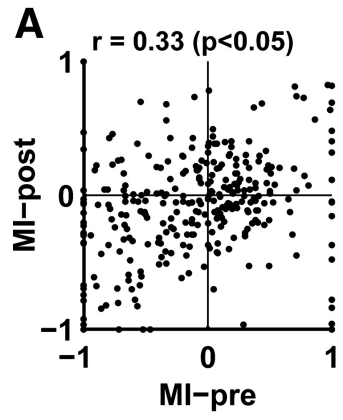

D

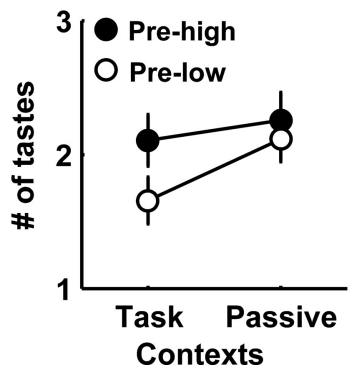

B

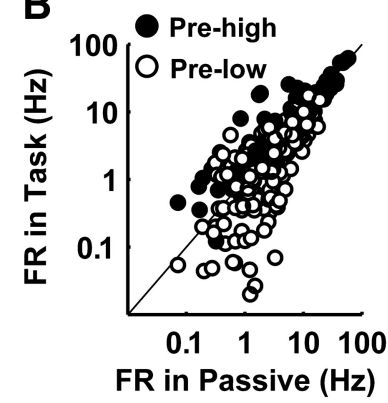

E

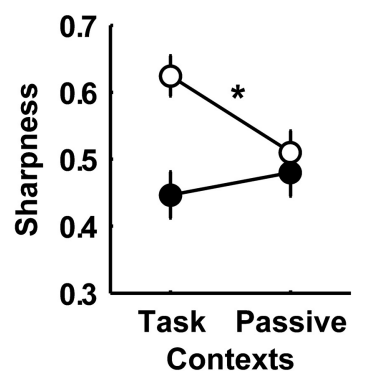

C

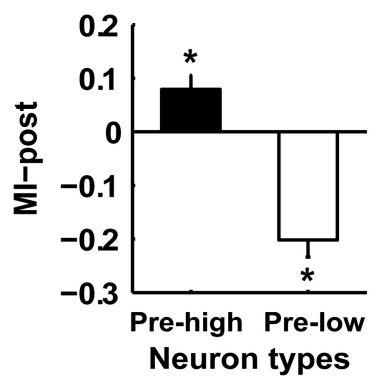

$\mathbf{F}$

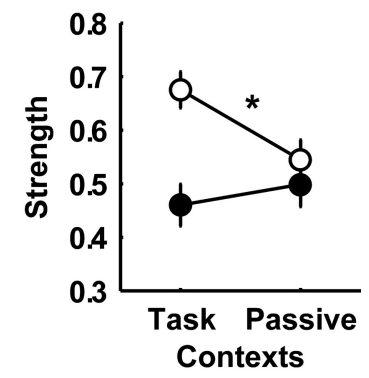

Figure 7. Taste response modulation is related to prestimulus activity changes. $\boldsymbol{A}$, The MI of taste responses is positively correlated with that in the prestimulus epoch. Each dot indicates a significant taste response ( $n=306$ responses pooled across tastes; $n=78,78,78$, and 72 responses to $\mathrm{N}, \mathrm{S}, \mathrm{C}$, and Q). Thirteen responses were removed because of 0 firings either in prestimulus or poststimulus epoch. Here, prestimulus Ml was computed for each response, using the mean firing rate in each taste (compare $\boldsymbol{B}$ ). $\boldsymbol{B}$, A between-context comparison of firing rates (FR) of taste responses in pre-high (filled circles; $n=135$ responses pooled across tastes from 47 pre-high neurons) and pre-low neurons (open circles; $n=180$ responses pooled across tastes from 70 pre-low neurons). Each neuron was classified as either pre-high or pre-low neurons via analysis of prestimulus MI (MI-pre; pre-high: Ml-pre $>0$ and pre-low: Ml-pre $<0$; 1 neuron was not classified as either type because the neuron failed to fire in prestimulus epoch). Significant taste responses in both types of neurons were plotted together, save for neurons that fired no action potentials. Here, Ml-pre was computed using the averaged firing rates across tastes for each neuron (compare $\boldsymbol{A}$ ). Many responses in pre-low neurons fall below the diagonal $(Y=X)$ line, indicating that task tends to decrease taste responses in pre-low neurons. Four responses were removed because the neurons failed to fire action potentials in either prestimulus or taste epochs. $\boldsymbol{C}$, The mean modulation indices of taste responses (Ml-post) in pre-high neuron ( $n=135$ responses pooled across tastes) and pre-low neurons ( $n=180$ responses pooled across tastes) are significantly higher and lower than 0 , respectively. The asterisk indicates $p<0.05$ by Wilcoxon's signed rank test with Bonferroni's correction. Four responses were removed because the neurons failed to fire action potentials in either prestimulus or taste epochs. $\boldsymbol{D}-\boldsymbol{F}$, A between-context comparison of the number of tastes inducing significant responses $(\boldsymbol{D})$, selectivity sharpness index $(\boldsymbol{E})$, and selectivity strength index $(\boldsymbol{F})$ in pre-high (filled circle) and pre-low neurons (open circle). $\boldsymbol{D}$, The context effect, but not the context-neuron type interaction is significant ( $p<0.05$ by 2W-RM-ANOVA; pre-high, $n=47$; pre-low, $n=70$ neurons). $\boldsymbol{E}, \boldsymbol{F}$, Asterisks indicate $p<0.05$ by simple main effects of context in pre-low neurons after significant context-neuron type interactions (pre-high, $n=47$; pre-low, $n=69 ; 1$ neuron was removed from pre-low neurons because it failed to fire action potentials in response to any tastes). Error bars indicate SEM.

of the five "worst" passive ensembles, the advantage of task over passive exceeded $10 \%$. As predicted from single neuron analyses (Fig. 7), the more pre-low neurons each population contained, the higher the improvement of score in task. The between- context difference of classification score was correlated with percentage of pre-low neurons contained in each population (i.e., [number of pre-low neurons/number of total neurons] $\times 100 \%)(r=0.60 ; p<0.05$; $n=14)$. We also computed classification scores for side-port-associated pairs of tastes (N-Q and $\mathrm{S}-\mathrm{C})$, as well as for palatability pairs (N-S and $\mathrm{C}-\mathrm{Q})$, and compared them. Scores for same-palatability pairs (task, $83 \pm 3 \%$; passive, $77 \pm 4 \%$; chance level was $50 \%$ in this case) was significantly higher than those for side-port-associated pairs (task, $78 \pm 3 \%$; passive, $72 \pm 3 \%)(p<$ $0.05,2 \mathrm{~W}-\mathrm{RM}-\mathrm{ANOVA}$ ), suggesting that association with side port was not a primary source of information represented across neurons in this experiment.

In summary, a rat called on to perform a sensory discrimination adjusts cortical networks such that neural responses provide higher quality information about stimuli.

\section{Discussion}

Because spontaneous activity affects sensory responses, it is natural to expect that an animal actively controls its prestimulus brain states to optimize sensory cortical processing for reliable stimulus identification (Desimone and Duncan, 1995; Arieli et al., 1996; Gilbert and Sigman, 2007; Fontanini and Katz, 2008; Haider and McCormick, 2009). Here, we show that in a discrimination task, rats modify both prestimulus and poststimulus activity, reducing suboptimal responses such that taste selectivity in GC is increased during stimulus discrimination, and also that the prestimulus and taste response modulations are intimately related. These results suggest that the control of prestimulus network state may allow higher-quality taste coding in the task context.

\section{Modulation of prestimulus activity}

Prestimulus modulation of cortical activity is a fundamental part of various cognitive processes, observable in attention (Luck et al., 1997; Reynolds et al., 2000) and motor preparation (Bastian et al., 2003; Churchland et al., 2006). In visual attention, such baseline activation may facilitate the subsequent processing of the attended feature of stimulus (Desimone and Duncan, 1995; Kastner and Ungerleider, 2000; Stokes et al., 2009). In the taste system, both selective attention and more general stimulus anticipation modulate prestimulus neural activity (Yamamoto et al., 1988; Ohgushi et al., 2005; Stapleton et al., 2006, 2007; Gutierrez et al., 2010) and subsequent taste responses in several brain areas including the primary taste cortex (Nitschke et al., 2006; Grabenhorst and Rolls, 2008). 
When animals can predict upcoming taste identity, prestimulus GC activity codes information about the specific taste (Stapleton et al., 2007).

When rats were encouraged to identify stimulus in a discrimination task, spontaneous firing rates were specifically made more "quiet," with rates below $\sim 3 \mathrm{~Hz}$ downregulated (often followed by decreases in taste response amplitudes) (see below). Trial-totrial firing rate variability before stimulus was reduced. In the passive context, the across-trial variability suddenly decreased after stimulus arrival, a phenomenon previously observed in several cortical areas (Churchland et al., 2010). In contrast, the variability dropped with the center port entry-approximately a second before stimulus delivery - in the task context, as previously reported in monkey premotor cortex during motor preparation (Churchland et al., 2006), but not, to our knowledge, during "sensory preparation." Reductions of across-trial variability are suggested to reflect the recruitment of brain area into the processing circuit, as indicated by the stimulusdriven reductions (Churchland et al., 2006, 2010). Thus, GC is likely to be involved in the process of discrimination even before the stimulus arrives.

Such reductions of across-trial variability may be interpreted as the conversion of network activity to a specific state (Churchland et al., 2006, 2010). State-to-state transitions are common in the dynamics of the gustatory and other systems (Jones et al., 2007; Churchland et al., 2010) (for review, see Buonomano and Maass, 2009). Theoretically, an initial state determines subsequent patterning in a complex dynamic system (Curto et al., 2009); network modeling studies demonstrate that an appropriate initial state facilitates effective stimulus processing (Martí et al., 2008; Miller and Katz, 2010). Thus, a rat may dynamically adjust the state of GC (and the larger gustatory system) to optimize subsequent stimulus processing within the discrimination task.

Relationship between prestimulus activity and taste response Although strong impacts of preexisting cortical activity on subsequent processing can be observed in anesthetized animals (Arieli et al., 1996; Castro-Alamancos, 2002; Murakami et al., 2005; Curto et al., 2009) (for review, see Haider and McCormick, 2009), it requires data from behaving animals to demonstrate that this could provide a possible mechanism of context-dependent modulation of sensory processing (Fontanini and Katz, 2006; Otazu et al., 2009) (for review, see Fontanini and Katz, 2008). In the rat GC, spike-shaped 7-12 Hz oscillations emerge in the ongoing LFP during reductions of an animal's arousal state, changing the taste responses such that information about taste palatability is highlighted (Fontanini and Katz, 2005, 2006; Tort et al., 2010). Selective attention to specific features of a visual scene, meanwhile, increases both the baseline activity and responses to attended stimuli, both in nonhuman primates (Luck et al., 1997; Reynolds et al., 2000) and humans (Kastner and Ungerleider, 2000; Stokes et al., 2009). In other modalities, modulations of sensory responses in "task versus passive" paradigms are accompanied by baseline activity changes (Krupa et al., 2004; Otazu et al., 2009).
Here, we extend these findings, showing that prestimulus modulations are reliably related to subsequent taste processing in single neurons. It has been shown that, during ingestive behavior, GC spontaneous firing is correlated with the magnitude of taste response in rat (Yamamoto et al., 1989) and taste selectivity in dog (Funakoshi and Ninomiya, 1983). Our results suggest that such strong relationship between the prestimulus and poststimulus activities may play a role in context-dependent modulations of taste coding. Similar correlation between prestimulus and poststimulus activity modulation in relation to behavioral context has been revealed in rat auditory system under a similar "task versus passive" paradigm (Otazu et al., 2009). In this work, increases in prestimulus thalamic activity were related to reductions of sensory responses in auditory cortex within the "task" context. Although many differences exist between that work and ours, both results suggest that control of prestimulus activity may improve subsequent sensory response modulation in certain situations.

In single neurons, decreases of prestimulus activity were followed by a suppression of taste responses, as if the modulations started before stimulus arrival, and simply carried over into the sensory processing. However, distinct spectral changes in LFPs between prestimulus and poststimulus epochs suggest mechanisms. It is suggested that beta range activity is related to relatively long-distance interactions, whereas gamma range activity reflects a function of local interactions (Kopell et al., 2000). Thus, one possibility is that the prestimulus modulation might be a part of relatively global interaction, whereas the poststimulus activity might be locally modified within a network in GC.

\section{Modulation of taste coding}

We also show that the firing rate changes of taste responses described above serve to improve stimulus coding in the period preceding the behavioral choice. Strong taste responses were (mostly) maintained, whereas weak, nonoptimal taste responses were reduced in size; inefficient responses were filtered out, resulting in an increase in taste selectivity-an increase that could be observed in pre-low neurons (Fig. 7), in the overall neural sample (Fig. 6), and in simultaneously recorded ensembles of neurons (Fig. 8). Thus, the modulation appears to enhance explicit representation of stimulus identity, a result that has not 
been reported in previous "task versus passive" studies using rats (Krupa et al., 2004; Fuentes et al., 2008; Otazu et al., 2009), probably for the purpose of sensory discrimination (although our rats produced too few error trials to permit direct tests of the relationship between taste response modulation and behavior). Similar sensory tuning has been observed in feature-based visual attention (Martinez-Trujillo and Treue, 2004) and under activated states in barrel cortex of anesthetized rat (Castro-Alamancos, 2002).

The taste response modulation was somehow scaled. The absolute amplitudes of task-related firing rate modulations were positively correlated with the mean firing rates of the responses [correlations $(r)$ : $0.50-0.67$ depending on taste] - modulation amplitudes become larger as responses become stronger. On average, taste response reductions were obvious in the weak activity of pre-low neurons (Figs. 4C,D, $7 B, C$ ) despite the small dynamic range. Therefore, this scaled modulation does not affect our interpretation that improved taste coding during task may be achieved by suppressing weak responses.

In addition to firing changes, both LFP evoked potential amplitudes and spectral power were lower during task. The evoked potential modulation suggests suppression of either the input to $\mathrm{GC}$, or the GC response to that input during task, consistent with the tendency toward decreased single-neuron taste responses. However, the link between LFP power and spike modulation is as of now indirect-that is, a causal link has yet to be established. Although the change of LFP power appears relatively modest, this may be related to the process of taste coding modulation in single-neuron level.

\section{Conclusions}

This study suggests a purpose for task-related prestimulus modulation: modulating the state of GC networks before stimulus delivery may enhance subsequent taste coding in a discrimination task. Although the data are currently correlative rather than causative - direct causal links among behavior, LFP, and spike firing modulations remain to be shown, and the biophysical mechanisms underlying relationship between prestimulus activity and subsequent sensory response modulation are beyond the scope of our investigation - this study provides novel findings supporting the idea that the control of ongoing activity may be one of mechanisms ensuring flexibility of sensory processing dependent on the specific needs of specific contexts (Desimone and Duncan, 1995; Arieli et al., 1996; Gilbert and Sigman, 2007; Fontanini and Katz, 2008; Haider and McCormick, 2009).

\section{References}

Accolla R, Carleton A (2008) Internal body state influences topographical plasticity of sensory representations in the rat gustatory cortex. Proc Natl Acad Sci U S A 105:4010-4015.

Arieli A, Sterkin A, Grinvald A, Aertsen A (1996) Dynamics of ongoing activity: explanation of the large variability in evoked cortical responses. Science 273:1868-1871.

Bastian A, Schöner G, Riehle A (2003) Preshaping and continuous evolution of motor cortical representations during movement preparation. Eur J Neurosci 18:2047-2058.

Buonomano DV, Maass W (2009) State-dependent computations: spatiotemporal processing in cortical networks. Nat Rev Neurosci 10:113-125.

Buzsáki G, Draguhn A (2004) Neuronal oscillations in cortical networks. Science 304:1926-1929.

Castro-Alamancos MA (2002) Role of thalamocortical sensory suppression during arousal: focusing sensory inputs in neocortex. J Neurosci 22:9651-9655.

Churchland MM, Yu BM, Ryu SI, Santhanam G, Shenoy KV (2006) Neural variability in premotor cortex provides a signature of motor preparation. J Neurosci 26:3697-3712.
Churchland MM, Yu BM, Cunningham JP, Sugrue LP, Cohen MR, Corrado GS, Newsome WT, Clark AM, Hosseini P, Scott BB, Bradley DC, Smith MA, Kohn A, Movshon JA, Armstrong KM, Moore T, Chang SW, Snyder LH, Lisberger SG, Priebe NJ, et al. (2010) Stimulus onset quenches neural variability: a widespread cortical phenomenon. Nat Neurosci 13:369-378.

Curto C, Sakata S, Marguet S, Itskov V, Harris KD (2009) A simple model of cortical dynamics explains variability and state dependence of sensory responses in urethane-anesthetized auditory cortex. J Neurosci 29:10600-10612.

de Araujo IE, Gutierrez R, Oliveira-Maia AJ, Pereira A Jr, Nicolelis MA, Simon SA (2006) Neural ensemble coding of satiety states. Neuron 51:483-494.

Desimone R, Duncan J (1995) Neural mechanisms of selective visual attention. Annu Rev Neurosci 18:193-222.

Fanselow EE, Nicolelis MA (1999) Behavioral modulation of tactile responses in the rat somatosensory system. J Neurosci 19:7603-7616.

Feierstein CE, Quirk MC, Uchida N, Sosulski DL, Mainen ZF (2006) Representation of spatial goals in rat orbitofrontal cortex. Neuron 51:495-507.

Fiser J, Chiu C, Weliky M (2004) Small modulation of ongoing cortical dynamics by sensory input during natural vision. Nature 431:573-578.

Foffani G, Moxon KA (2004) PSTH-based classification of sensory stimuli using ensembles of single neurons. J Neurosci Methods 135:107-120.

Fontanini A, Katz DB (2005) 7 to $12 \mathrm{~Hz}$ activity in rat gustatory cortex reflects disengagement from a fluid self-administration task. J Neurophysiol 93:2832-2840.

Fontanini A, Katz DB (2006) State-dependent modulation of time-varying gustatory responses. J Neurophysiol 96:3183-3193.

Fontanini A, Katz DB (2008) Behavioral states, network states, and sensory response variability. J Neurophysiol 100:1160-1168.

Freedman DJ, Riesenhuber M, Poggio T, Miller EK (2006) Experiencedependent sharpening of visual shape selectivity in inferior temporal cortex. Cereb Cortex 16:1631-1644.

Fuentes RA, Aguilar MI, Aylwin ML, Maldonado PE (2008) Neuronal activity of mitral-tufted cells in awake rats during passive and active odorant stimulation. J Neurophysiol 100:422-430.

Funakoshi M, Ninomiya Y (1983) Relations between the spontaneous firing rate and taste responsiveness of the dog cortical neurons. Brain Res 262:155-159.

Furuyashiki T, Holland PC, Gallagher M (2008) Rat orbitofrontal cortex separately encodes response and outcome information during performance of goal-directed behavior. J Neurosci 28:5127-5138.

Gilbert CD, Sigman M (2007) Brain states: top-down influences in sensory processing. Neuron 54:677-696.

Grabenhorst F, Rolls ET (2008) Selective attention to affective value alters how the brain processes taste stimuli. Eur J Neurosci 27:723-729.

Grossman SE, Fontanini A, Wieskopf JS, Katz DB (2008) Learning-related plasticity of temporal coding in simultaneously recorded amygdalacortical ensembles. J Neurosci 28:2864-2873.

Gutierrez R, Simon SA, Nicolelis MA (2010) Licking-induced synchrony in the taste-reward circuit improves cue discrimination during learning. J Neurosci 30:287-303.

Haider B, McCormick DA (2009) Rapid neocortical dynamics: cellular and network mechanisms. Neuron 62:171-189.

Halpern BP, Tapper DN (1971) Taste stimuli: quality coding time. Science 171:1256-1258.

Ito S, Ogawa H (1994) Neural activities in the fronto-opercular cortex of macaque monkeys during tasting and mastication. Jpn J Physiol 44:141-156.

Jones LM, Fontanini A, Sadacca BF, Miller P, Katz DB (2007) Natural stimuli evoke dynamic sequences of states in sensory cortical ensembles. Proc Natl Acad Sci U S A 104:18772-18777.

Kastner S, Ungerleider LG (2000) Mechanisms of visual attention in the human cortex. Annu Rev Neurosci 23:315-341.

Katz DB, Simon SA, Nicolelis MA (2001a) Dynamic and multimodal responses of gustatory cortical neurons in awake rats. J Neurosci $21: 4478-4489$.

Katz DB, Simon SA, Nicolelis MA (2001b) Electrophysiological studies of gustation in awake rats. In: Methods and frontiers in the chemical senses (Simon SA, Nicolelis MA, eds), pp 339-357. Boca Raton, FL: CRC.

Kopell N, Ermentrout GB, Whittington MA, Traub RD (2000) Gamma 
rhythms and beta rhythms have different synchronization properties. Proc Natl Acad Sci U S A 97:1867-1872.

Krupa DJ, Wiest MC, Shuler MG, Laubach M, Nicolelis MA (2004) Layerspecific somatosensory cortical activation during active tactile discrimination. Science 304:1989-1992.

Luck SJ, Chelazzi L, Hillyard SA, Desimone R (1997) Neural mechanisms of spatial selective attention in areas V1, V2, and V4 of macaque visual cortex. J Neurophysiol 77:24-42.

MacDonald CJ, Meck WH, Simon SA, Nicolelis MA (2009) Taste-guided decisions differentially engage neuronal ensembles across gustatory cortices. J Neurosci 29:11271-11282.

Martí D, Deco G, Mattia M, Gigante G, Del Giudice P (2008) A fluctuationdriven mechanism for slow decision processes in reverberant networks. PLoS One 3:e2534.

Martinez-Trujillo JC, Treue S (2004) Feature-based attention increases the selectivity of population responses in primate visual cortex. Curr Biol 14:744-751.

Miller P, Katz DB (2010) Stochastic transitions between neural states in taste processing and decision-making. J Neurosci 30:2559-2570.

Murakami M, Kashiwadani H, Kirino Y, Mori K (2005) State-dependent sensory gating in olfactory cortex. Neuron 46:285-296.

Nitschke JB, Dixon GE, Sarinopoulos I, Short SJ, Cohen JD, Smith EE, Kosslyn SM, Rose RM, Davidson RJ (2006) Altering expectancy dampens neural response to aversive taste in primary taste cortex. Nat Neurosci 9:435-442.

Ohgushi M, Ifuku H, Ito S, Ogawa H (2005) Response properties of neurons to sucrose in the reward phase and the areal distribution in the monkey fronto-operculuro-insular and prefrontal cortices during a taste discrimination GO/NOGO task. Neurosci Res 51:253-263.

Otazu GH, Tai LH, Yang Y, Zador AM (2009) Engaging in an auditory task suppresses responses in auditory cortex. Nat Neurosci 12:646-654.

Paxinos G, Watson C (1998) The rat brain in stereotaxic coordinates, Ed 4. San Diego: Academic.

Petersen CC, Hahn TT, Mehta M, Grinvald A, Sakmann B (2003) Interac- tion of sensory responses with spontaneous depolarization in layer $2 / 3$ barrel cortex. Proc Natl Acad Sci U S A 100:13638-13643.

Rainer G, Asaad WF, Miller EK (1998) Selective representation of relevant information by neurons in the primate prefrontal cortex. Nature 393:577-579.

Reynolds JH, Pasternak T, Desimone R (2000) Attention increases sensitivity of V4 neurons. Neuron 26:703-714.

Roesch MR, Taylor AR, Schoenbaum G (2006) Encoding of timediscounted rewards in orbitofrontal cortex is independent of value representation. Neuron 51:509-520.

Rolls ET, Scott TR, Sienkiewicz ZJ, Yaxley S (1988) The responsiveness of neurones in the frontal opercular gustatory cortex of the macaque monkey is independent of hunger. J Physiol 397:1-12.

Smith-Swintosky VL, Plata-Salaman CR, Scott TR (1991) Gustatory neural coding in the monkey cortex: stimulus quality. J Neurophysiol 66:1156-1165.

Stapleton JR, Lavine ML, Wolpert RL, Nicolelis MA, Simon SA (2006) Rapid taste responses in the gustatory cortex during licking. J Neurosci 26:4126-4138

Stapleton JR, Lavine ML, Nicolelis MA, Simon SA (2007) Ensembles of gustatory cortical neurons anticipate and discriminate between tastants in a single lick. Front Neurosci 1:161-174.

Stokes M, Thompson R, Nobre AC, Duncan J (2009) Shape-specific preparatory activity mediates attention to targets in human visual cortex. Proc Natl Acad Sci U S A 106:19569-19574.

Tort AB, Fontanini A, Kramer MA, Jones-Lush LM, Kopell NJ, Katz DB (2010) Cortical networks produce three distinct $7-12 \mathrm{~Hz}$ rhythms during single sensory responses in the awake rat. J Neurosci 30:4315-4324.

Yamamoto T, Matsuo R, Kiyomitsu Y, Kitamura R (1988) Sensory inputs from the oral region to the cerebral cortex in behaving rats: an analysis of unit responses in cortical somatosensory and taste areas during ingestive behavior. J Neurophysiol 60:1303-1321.

Yamamoto T, Matsuo R, Kiyomitsu Y, Kitamura R (1989) Taste responses of cortical neurons in freely ingesting rats. J Neurophysiol 61:1244-1258. 